\title{
Rotational and Vibrational bands in ${ }^{108} \mathbf{P d}$
}

\author{
J.A. Alcántara-Núñez, J.R.B. Oliveira, E.W. Cybulska, N.H. Medina, \\ M.N. Rao, R.V. Ribas, M.A. Rizzutto,W.A. Seale, and F. Falla-Sotelo \\ Instituto de Física, Universidade de São Paulo, C.P. 66318, São Paulo, SP, 05315-970, Brazil
}

Received on 3 November, 2003

\begin{abstract}
The ${ }^{108} \mathrm{Pd}$ nucleus has been studied with the ${ }^{100} \mathrm{Mo}\left({ }^{11} \mathrm{~B}, p 2 n \gamma\right)$ reaction at $43 \mathrm{MeV}$ incident energy. $\gamma-\gamma-t$, $\gamma-\gamma-$ charged particle coincidences and directional correlation ratios were measured using the $\gamma$ spectrometer formed by four Compton suppressed HPGe detectors and a $4 \pi$ charged-particle ancillary detector system. The struture of the bands was interpreted within the framework of the cranked shell model and total Routhian calculations.
\end{abstract}

The nuclei around $Z=44$ and $N=60$ are susceptible to dramatic changes in shape with the addition or subtraction of a small number of nucleons [1]. In the region of $\mathrm{Pd}$ the rotational alignment of proton $\left(g_{9 / 2}\right)$ and neutron $\left(h_{11 / 2}\right)$ intruder orbitals can give rise to a significant increase in aligned angular momentum, resulting in a variety of collective behaviors. Previous experiments on ${ }^{100-106} \mathrm{Pd}$ show that the majority of states populated by $(\mathrm{HI}, x n)$ reaction are members of decoupled collective bands built on quasineutron states [2-4].

On the other hand, the nuclei in the region of $\mathrm{Pd}$ and $\mathrm{Ru}$ are characterized by vibrational structures and modest deformation at low energies because they are near the $Z=50$ shell closure. It can be commonly mentioned that the structure of their lowest-lying states is dominated by vibrational degrees of freedom as reflected in the typical vibrational $0^{+}$, $2^{+}$and $4^{+}$triplet of states at about twice the energy of the first $2^{+}$state [5]. This statement is corroborated by empirical analysis E-GOS, proposed by P. Regan [6].

In this work, we present the results of an investigation of ${ }^{108} \mathrm{Pd}$ with the ${ }^{100} \mathrm{Mo}\left({ }^{11} \mathrm{~B}, p 2 n \gamma\right)$ reaction at $43 \mathrm{MeV}$ beam energy. The beam was provided by the Pelletron Tandem Accelerator of the University of São Paulo. The target used was sufficiently thick in order to stop the recoils. Gamma rays and charged particles have been detected using the SACI-PERERE array. SACI [7] (Sistema Ancilar de Cintiladores) is a $4 \pi$ charged particle telescope system consisting of 11 plastic phoswich scintillators, disposed in the geometry of a dodecahedron, which enabled the selection of the evaporated charged particle fold in coincidence with the observed $\gamma$-rays. PERERE [8] (Pequeno Espectrômetro de Radiação Eletromagnética com Rejeição de Espalhamento) is the $\gamma$-ray spectrometer consisting of 4 HPGe detectors with BGO Compton-shields. A partial selection of specific channels can be made by detecting and identifying some or all of the evaporating particles in coincidence with $\gamma$-rays. Events were collected when at least 2 Ge detectors fired in coincidence. A total of $85 \times 10^{6}$ Compton-suppressed events was collected. The data have been sorted into symmetrized $\gamma-\gamma$ and $\gamma-\gamma-$ charged particle matrices. The $\gamma-$ ray transitions belonging to ${ }^{108} \mathrm{Pd}$ were identified by setting gates on charged particle fold $1 p$ (see Fig. 1). The data were analysed using the VPAK [9] and RADWARE [10] spectrum analysis codes.

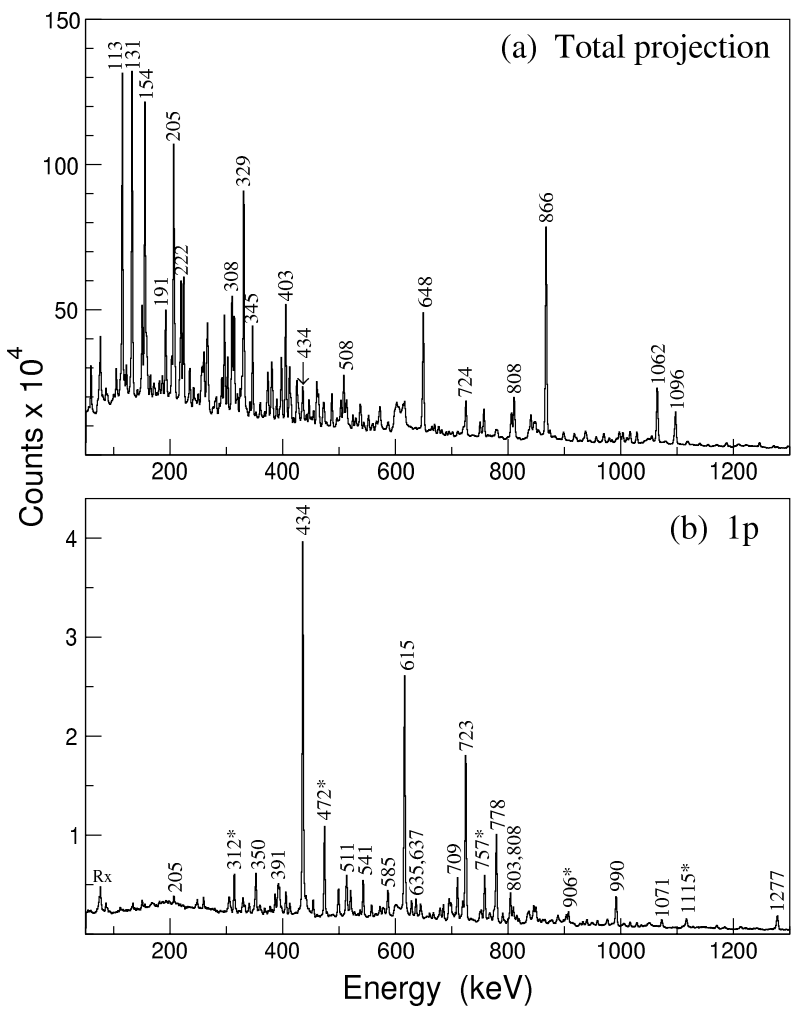

Figure 1. Gamma-ray spectra (a) Total projection and (b) gated with particle fold $1 p$. In $(a)$ most lines are from ${ }^{107,108} \mathrm{Ag}$ and in (b) from ${ }^{108} \mathrm{Pd}$. The transitions marked with asterisks belong to ${ }^{107} \mathrm{Pd}$.

Previous to the present work, only three bands were known $[11,12]$. Two new rotational structures at relatively high excitation energy were identified (labelled $3 b$ and 4 in Fig. 2). 


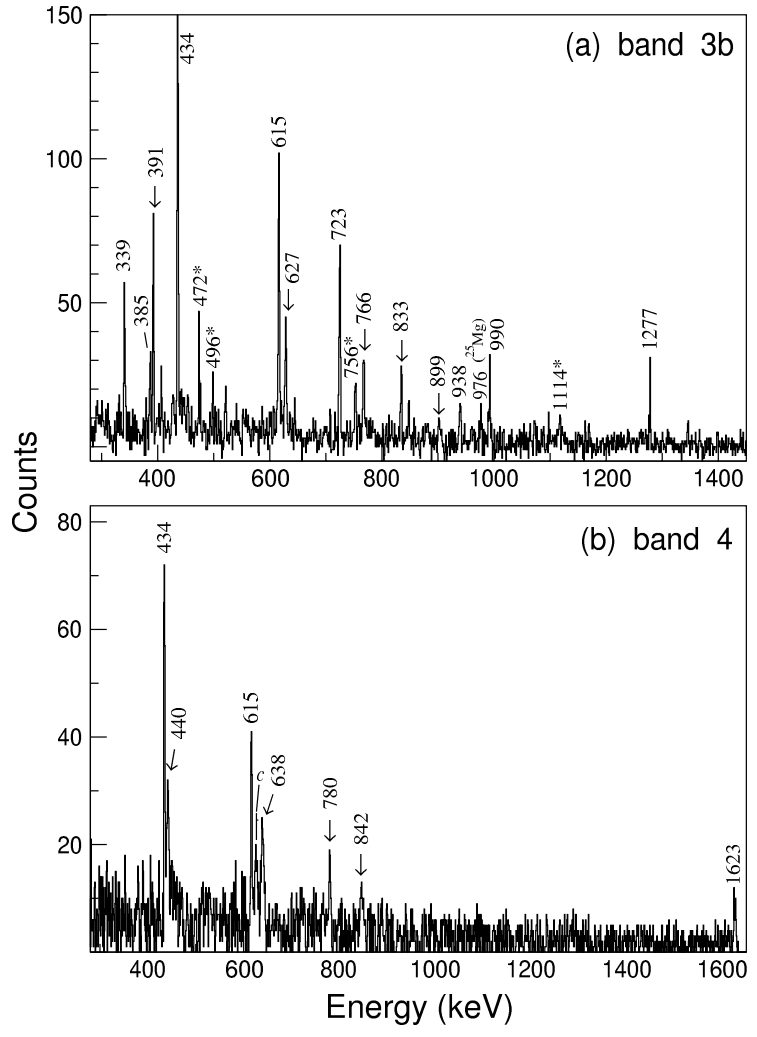

Figure 2. Gamma-ray spectra from the $1 p$ gated matrix. In band transitions are indicated by arrows. Sum of gates on (a) 627, 766 and $833 \mathrm{keV}$ transitions belonging to band $3 \mathrm{~b}$ and (b) 440, 638 and $1623 \mathrm{keV}$ transitions belonging to band 4 , in ${ }^{108} \mathrm{Pd}$. The transitions marked with asterisks belong to ${ }^{107} \mathrm{Pd}$ and the transition marked with "c" is an unidentified contaminant.

One of these new bands (3b) corresponds to the unfavored signature of $\nu\left(h_{11 / 2} \otimes g_{7 / 2}\right)$ configuration. The favored signature band (3a) was identified in previous work [11]. TRS calculations (Fig. 3(a)) predict a nearly axial symetry with $\beta_{2} \approx 0.19$ and $\gamma \approx 4^{\circ}$ as deformation parameters.

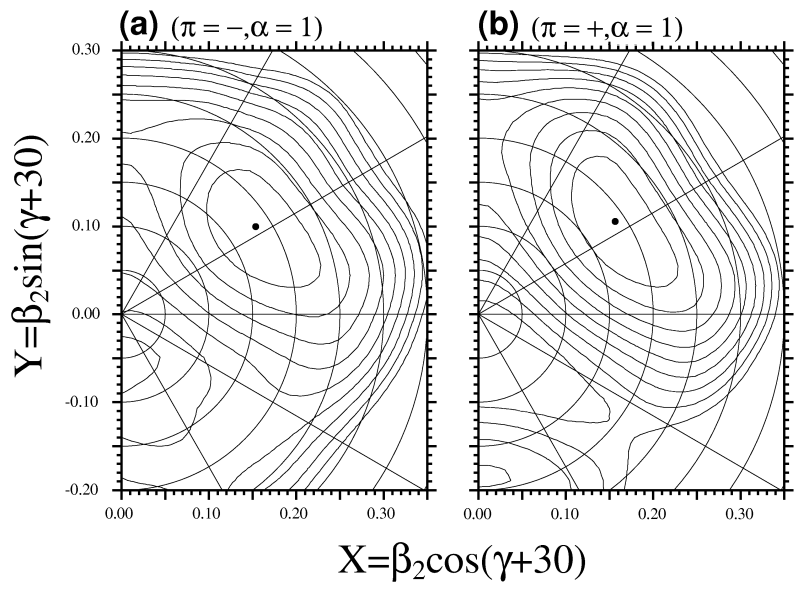

Figure 3. Total Routhian Surface calculations (a) $\nu h_{11 / 2} \otimes$ $\left(g_{7 / 2}, d_{5 / 2}\right)$ and (b) excited $\left(\nu h_{11 / 2}\right)^{2}$ configurations at $\hbar \omega=0.25$ $\mathrm{MeV}$ in ${ }^{108} \mathrm{Pd}$. The thick dot indicates the position of the equilibrium deformation.

The quasiparticle Routhians, calculated with the CSM, based on a deformed Woods-Saxon potential including pair- ing interaction, show that the $\nu\left(h_{11 / 2} \otimes g_{7 / 2}\right)$ configuration has a low excitation energy (for two quasineutrons) with an aligned angular momentum $i_{x} \approx 8 \hbar$ and signature splitting $\Delta e^{\prime} \approx 100 \mathrm{keV}$. This is in agreement with the experimental Routhians (see Fig. 4) for bands 3a and $3 b$.

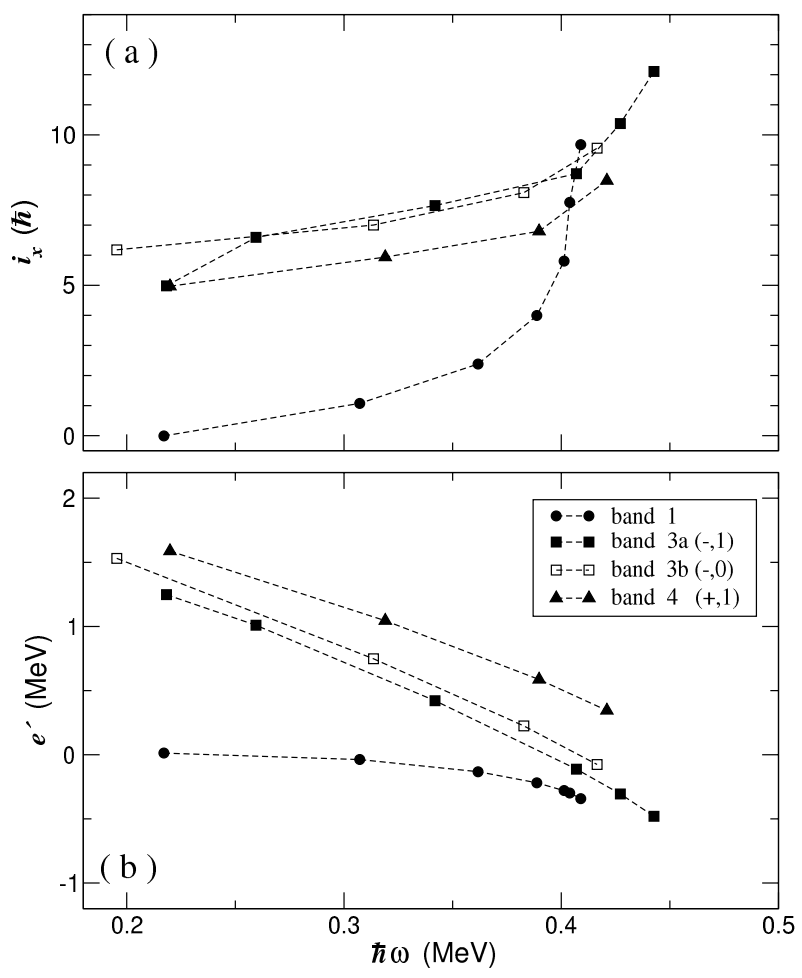

Figure 4. Experimental quasiparticle $(a)$ Aligned angular momentum $i_{x}$ and $(b)$ Routhians as a function of the rotational frequency $\hbar \omega$ for positive and negative parity bands in ${ }^{108} \mathrm{Pd}$.

TRS (Fig. 3(b)) and CSM calculation show that another configuration with positive parity and lower excitation energy is the excited $\left(\nu h_{11 / 2}\right)^{2}$ with $\alpha=1$ as a favored signature and an aligned angular momentum $i_{x} \approx 7 \hbar$. In this configuration the first neutron crossing (at $\hbar \omega \approx 0.3 \mathrm{MeV}$ ) is blocked. Experimentally, band 4 presents similar characteristics with the assumption of a $\mathrm{I}^{\pi}=\left(5^{+}\right)$state as a band head. For this reason we assign to band 4 the excited $\left(\nu h_{11 / 2}\right)^{2}$ configuration.

A low spin $\gamma$-vibrational band, with some states previously observed by L. Svensson [5], was identified and extended up to $\mathrm{I}^{\pi}=10^{+}$.

\section{Acknowledgments}

We thank the technical staff of the Pelletron Tandem Accelerator of the University of São Paulo. This work was partially supported by the Fundação de Amparo à Pesquisa do Estado de São Paulo (FAPESP) and the Conselho Nacional de Desenvolvimento Científico e Tecnológico (CNPq), Brazil. 


\section{References}

[1] Nuclear Structure of the Zirconium Region, edited by J. Eberth, R. Meyer, and K. Sistemich (Springer-Verlag, Berlin, 1988).

[2] J.A. Grau, L.E. Samuelson, F.A. Rickey, P.C. Simms, and G.J. Smith, Phys. Rev. C14, 2297 (1976).

[3] Dan Jerrestam, W. Klamra, B. Fogelberg, R. Bark, A. Gizon, J. Gizon, E. Iddeguchi，S. Mitarai，M. Piiarinen, and G. Sletten, Nucl. Phys. A603, 203 (1996).

[4] G.E. Perez, et al., Nucl. Phys. A686, 41 (2001).

[5] L.E. Svensson, C. Fahlander, L. Hasselgren, A. Bäcklin, L.Westerberg, D. Cline, T. Czosnyka, C.Y. Wu, R.M. Diamond, and H. Klue, Nucl. Phys. A584, 547 (1995).

[6] P.H. Regan, C.W. Beausang, N.V. Zamfir, R.F. Casten, Jingye Zhang, A.D. Yamamoto, M.A. Caprio, G. Gürdal, A.A. Hecht, C. Hutter, R. Krücken, S.D. Langdown, D.A. Meyer, and J.J.Ressler, Phys. Rev. Lett. Vol90, 152502 (2003).

[7] J. A. Alcántara-Núñez, J.R.B. Oliveira, E.W. Cybulska, N.H. Medina, M.N. Rao, R.V. Ribas, M.A. Rizzutto, W.A. Seale, F. Falla-Sotelo, F.R. Espinoza-Qiñones, and C. Tenreiro, Nucl. Instrum. Methods Phys. Res. A497, 429 (2003).

[8] R.V. Ribas, J.R.B. Oliveira, E.W. Cybulska, M.N. Rao, W.A. Seale, M.A. Rizzutto, and N.H. Medina, Ann. Rep. of the Nucl. Phys. Dept., Institute of Physics, University of São Paulo, p. 63 (1996).

[9] W. M. Milner - Oak Ridge National Laboratory (1986).

[10] D. Radford, Nucl. Instrum. Methods Phys. Res. A361, 297 (1995).

[11] K.R Pohl, P.H. Regan, J.E. Bush, P.E. Raines, D.P. Balamuth, D. Ward, A. Galindo-Uribarri, V.P. Janzen, S.M. Mullins, and S. Pilitte, Phys. Rev. C53, 2682 (1996).

[12] P.H. Regan, T.M. Menezes, C.J. Pearson, W. Galletly, C.S. Purry, P.M Walker, S. Juutinen, R. Julin, K. Helariutta, A. Savelius, P. Jones, P. Jämsen, M. Muikku, P. A. Butler, G. Jones, and P. Greenlees, Phys. Rev. C33, 2305 (1997). 